\title{
Valoración de buenas prácticas docentes a través de observación sistemática*
}

\section{Assessing of Good Teaching Practicesthrough Systematic Observation}

Enviado: 16 de septiembre de 2014 |Revisado: 3 de julio de 2015 | Aceptado: 28 de julio de 2015

\author{
Matilde Candelaria Díaz HeRnándeZ ** \\ MARÍA DE ÁFrICA BORGES DEL ROSAL *** \\ Universidad de La Laguna, España \\ María de los Dolores VAladez SierRA **** \\ ROGELIO ZAMBRANO GUZMÁN ****** \\ Universidad de Guadalajara, México
}

doi:10.11144/Javeriana.upsy14-3.vbpd

Para citar este artículo: Díaz, M. C.; Borges, A. Valadez, M. D.; Zambrano, R. (2015). Valoración de buenas prácticas docentes a través de observación sistemática. Universitas Psychologica, 14(3), 913-922. http://dx.doi.org/10.11144/Javeriana.upsy14-3.vbpd

* Artículo de investigación

** Profesora del Departamento de Psicología Cognitiva, Social y Organizacional. Universidad de La Laguna, Campus de Guajara, Tenerife, España. Correo electrónico:: macadine@ull.edu.es

**** Profesora del Departamento de Psicología Clínica, Psicobiología y Metodología, Universidad de La Laguna, Campus de Guajara, Tenerife, España. Correo electrónico: aborges@ull.edu.es

****** Profesora del Departamento de Psicología Aplicada, Centro Universitario Ciencias de la Salud, Universidad de Guadalajara, Jalisco, México. Correo electrónico: doloresvaladez@yahoo.com.mx

******* Profesor del Departamento de Psicología Aplicada, Centro Universitario Ciencias de la Salud, Universidad de Guadalajara, Jalisco, México. Correo electrónico: rogelio@cucs.udg.mx

\section{RE S U MEN}

Las buenas prácticas docentes requieren del diseño de distintas formas de medición al ser un tema de especial relevancia para determinar la calidad de la educación superior. Una gran parte de las buenas prácticas corresponden a comportamientos desarrollados por el profesor en el aula de clase, lo que se puede medir y analizar mediante metodología observacional. Se seleccionaron diez profesores de la Universidad de La Laguna (España) y siete de la Universidad de Guadalajara (México), codificando sus comportamientos mediante una escala de calificación y los patrones comportamentales de interacción con el alumnado mediante el Protocolo de Observación de Funciones Docentes (PROFUNDO_UNI). El análisis secuencial de retardos extrajo patrones significativos relacionados con la retroalimentación a las preguntas del alumnado, el refuerzo dado y el fomento a la participación de los estudiantes.

Palabras clave

buenas prácticas docentes; evaluación del docente; metodología observacional

\section{A B S T R A C T}

The best teaching practices, an issue of particular relevance in determining the quality of higher education, requires different forms of measuring design. A big part of good practice in teacher performance corresponds to behaviors developed by the teacher in the classroom, which can be measured and analyzed using observational methodology. The study evaluates ten teachers from the University of La Laguna (Spain) and seven from Guadalajara (Mexico). Their behavior was coded using a rating scale and the behavioral interaction patterns with students were coded using the Observational Protocol of Teaching Functions (Protocolo de Observación de Funciones Docentes, PROFUNDO_UNI). The lag sequential analysis extracted significant patterns related to the feedback given to questions from the students, the reinforcement and the encouragement given to the students for their participation.

Keywords

best practices; teacher evaluation; observational methodology 


\section{Introducción}

El término buenas prácticas se ha acuñado para explicar la adecuada actuación del profesional en diversos ámbitos laborales, aplicándose en actividades muy diversas y adquiriendo diversos usos. Alude a desarrollar una actividad, normalmente innovadora, que se ha experimentado y evaluado, comprobándose su éxito (Cid-Sabucedo, PérezAbellás, \& Zabalza, 2009).

En el ámbito educativo las buenas prácticas educativas se entienden como las acciones relacionadas con el cuidado, la enseñanza y la orientación de otras personas, configurándose como saberes estratégicos, conocimientos de saberes y motivaciones (Gimeno, 1999; citado en Gaitán, Campos, García, Granados, Jaravillo \& Manquera, 2005).

Bain (2006) realizó un interesante estudio, donde se analizan y recogen las características observables y no observables de las buenas prácticas de profesores considerados por el autor como extraordinarios por ser exitosos ayudando a sus estudiantes a aprender, ejerciendo una influencia positiva en su manera de pensar, actuar y sentir. Se les observó en el aula y en el laboratorio, se realizaron entrevistas con los profesores seleccionados y con su alumnado, se consultaron varios documentos (programas, materiales del curso, tareas, etc.) y los resultados sobre las valoraciones de los estudiantes. Como resultado del estudio, se contemplan como buenas prácticas aspectos tales como lo que sabe el profesor y cómo prepara las clases, la forma en que dicta clases, la actitud y el respeto hacia el alumnado, su estilo -estimulando, interesando e implicando al estudiantado, así como fomentando su participación y respondiendo a las preguntas que se le plantean-. También se considera importante la forma de evaluar, en la medida que se considera como una parte del aprendizaje.

Marqués (2002) distingue tres momentos en la aplicación de las buenas prácticas docentes determinadas por el proceder del educador: a) antes de la intervención docente, momento en el que se toman en cuenta características grupales e individuales de los estudiantes, se plantean los objetivos, los contenidos concretos a tratar, el diseño de estrategias para la realización de actividades y las metodologías docentes a emplear; b) en la intervención docente, que puede ser lineal, con la exposición del profesor, tutoría o asesoramiento personalizado o bien poligonal o en red, tales como el trabajo en grupo o las discusiones entre todos en clase; y c) después de la intervención docente, cuando el profesor procede a elaborar una reflexión para la mejora de futuras intervenciones educativas.

Imbernón (2013) señala como buenas prácticas docentes el estar de pie durante la exposición de contenidos, aproximarse al alumnado, hablar con un ritmo adecuado (ni lento, ni rápido), hacer las anotaciones en la pizarra con letra clara y visible - preferentemente en mayúsculas- para garantizar su lectura desde cualquier punto del aula, borrando lo que se haya escrito anteriormente para que no se mezclen los contenidos previos con los nuevos. Las presentaciones multimedia deben tener poco texto, letra y colores visibles, de forma que permitan su lectura y no dependan de la luminosidad de la sala. Son indicadores de malas prácticas sentarse en la mesa, dar la espalda al alumnado -ya sea por pasear mientras se explica o por hablar escribiendo en la pizarra, ya que dificulta ser escuchado-,leer, tanto de un libro como de las presentaciones multimedia y presentar una indumentaria desaliñada.

Algunos aspectos de buenas prácticas pueden captarse a través de instrumentos creados para tal efecto (Gaitán, Campo, García, Granados, Jaramillo, \& Panqueva, 2005). Cuando se trata de analizar conductas la metodología observacional es la más indicada (Anguera, 2008), ya que permite establecer la existencia de comportamientos y evaluar la interacción entre agentes (en este caso, el profesor y su alumnado), esta se puede medir con un análisis secuencial (Bakeman \& Quera, 1996)

El objetivo de la presente investigación es describir indicadores observacionales de buenas prácticas educativas, así como patrones comportamentales exhibidos en el aula mientras el profesorado desarrolla su labor docente. 


\section{Método}

\section{Participantes}

Mediante muestreo intencional de casos típicos se seleccionaron ocho profesores y dos profesoras de la Universidad de La Laguna (ULL), dos por cada rama del conocimiento (Arquitectura e Ingeniería; Artes y Humanidades; Ciencias; Ciencias Jurídicas y Sociales; Ciencias de la Salud), con edades comprendidas entre 49 y 61 años (media $=52.8$; $\mathrm{DT}=4.8)$ que imparten asignaturas de diverso tipo (básicas de rama, obligatorias u optativas), con una experiencia docente entre 15 y 32 años (media= 23.1; DT= 4.9). Se seleccionaron también cinco varones y dos mujeres profesores de la carrera de Psicología de la Universidad de Guadalajara (UdG), México, dos de ellos más jóvenes ( 25 y 27 años) y con una experiencia docente de menos de un año, los restantes se encuentran en un rango de edad entre 50 y 57 (media $=54$; DT $=3.3$ ) y una expe- riencia docente entre 20 y 33 años (media= 25.6; $\mathrm{DT}=5.6$ ), dictando asignaturas obligatorias que se cursan en quinto semestre de la carrera.

\section{Instrumentos}

\section{Escala de calificación}

Para el registro de los recursos utilizados y las conductas desarrolladas en el aula se utilizó una escala de calificación donde el observador marcaba la aparición de un recurso utilizado (por ejemplo, presentaciones multimedia o uso de pizarra) o de la conducta realizada (por ejemplo, postura al dar la clase, llamar por su nombre al alumnado). Los aspectos recogidos se presentan en la Tabla 2.

\section{Instrumento de observación}

Para la codificación de las sesiones grabadas y con objeto de obtener patrones de comportamiento,

\section{TABLA 1.}

Protocolo de observación de funciones docentes en universidad (PROFUNDO UNI), versión 3

\begin{tabular}{|c|c|c|}
\hline Funciones docentes & Códigos & \\
\hline \multirow{3}{*}{$\begin{array}{l}\text { Organización: Estructuración del contexto, disposición del material } \\
\text { didáctico, establecer la normativa, determinar el tiempo de las tareas, etc. }\end{array}$} & Planificación académica & $\mathrm{PA}$ \\
\hline & Del contexto & $\mathrm{OD}$ \\
\hline & Del alumnado & $\mathrm{OA}$ \\
\hline \multirow{2}{*}{$\begin{array}{l}\text { Comunicabilidad docente: Explicación de contenidos de forma } \\
\text { comprensible para el alumnado }\end{array}$} & Explicación del profesor & EP \\
\hline & Respuesta al alumno & RA \\
\hline \multirow{2}{*}{ Motivadora: Estimular el aprendizaje del alumno. } & Refuerzo & $\mathrm{RF}$ \\
\hline & Fomento participación & FP \\
\hline $\begin{array}{l}\text { Control comportamental o regulación del grupo: Gestión del orden en el } \\
\text { aula }\end{array}$ & Contingencia negativa & $\mathrm{CN}$ \\
\hline \multirow{2}{*}{$\begin{array}{l}\text { Orientación y asesoramiento: Directrices para el desempeño de las tareas del } \\
\text { alumnado }\end{array}$} & Guía & GU \\
\hline & Revisión no verbal & $\mathrm{RN}$ \\
\hline \multirow{2}{*}{$\begin{array}{l}\text { Interacción del estudiante: Intervenciones del alumnado por iniciativa } \\
\text { propia o respondiendo al docente }\end{array}$} & Participación del estudiantado & $\mathrm{PE}$ \\
\hline & Responde al profesor & $\mathrm{RP}$ \\
\hline $\begin{array}{l}\text { Interacción disruptiva: Conducta del alumnado que impide el desarrollo de } \\
\text { la clase }\end{array}$ & Disrupciones del alumnado & $\mathrm{DA}$ \\
\hline \multirow{2}{*}{$\begin{array}{l}\text { Interacción general: Comentarios con contenidos ajenos a las explicaciones } \\
\text { de esa clase }\end{array}$} & I. Generales del profesor & PIG \\
\hline & I. Generales del alumnado & $\mathrm{AIG}$ \\
\hline \multirow{3}{*}{ Categorías instrumentales } & Otros comportamientos & $\mathrm{X}$ \\
\hline & Inobservable & $\mathrm{Y}$ \\
\hline & El profesor sale del aula & Z \\
\hline
\end{tabular}

Fuente: elaboración propia 
se utilizó el protocolo de observación de funciones docentes en universidad (PROFUNDO_Uni, v3; Rodríguez, Cadenas \& Díaz, 2011), diseñado para evaluar la conducta del profesorado en su desempeño docente en el aula.

En la Tabla 1 se presentan las funciones y su definición, la operativización en códigos directamente observables y la abreviatura de codificación. En la categoría instrumental se recogen conductas que no corresponden a funciones docentes: Otros comportamientos: $\mathrm{X}$, conductas que realiza fuera de cámara, Conducta Inobservable: Y, y cuando se ausenta, 'Profesor sale del aula': Z (Rodríguez, Cadenas \& Díaz, 2011).

Instrumentos de registro

Las sesiones se grabaron en la ULL con dos cámaras de vídeo, SONY DCR-SR58E y JVC GZ MG750, y en la UdG con una SONY HDR CX100 Handycam.

\section{Metodología y diseño}

Se empleó una metodología observacional con un diseño nomotético de seguimiento y multidimensional (Anguera, Blanco \& Losada, 2001).

\section{Procedimiento}

Siguiendo la normativa en materia de protección de datos de ambos países, profesores y estudiantes aceptaron ser grabados con consentimiento informado. Antes de la codificación de datos se entrenó a los observadores según un procedimiento estandarizado (Cadenas, Rodríguez \& Díaz, 2012; Rodríguez-Naveiras, 2011), diez observadores en la ULL, nueve mujeres y un varón, de entre 23 y 40 años y ocho en la $U d G$, seis mujeres y dos varones, con edades entre 22 y 34 años, siendo sus titulaciones Máster o Licenciatura en Psicología o en Educación Infantil y estudiantes de Maestría, Psicología y Logopedia.

De cada profesor se grabaron de 10 a 12 horas impartiendo docencia. En el profesorado de la ULL se incluyeron clases prácticas. Para contrarrestar la posible reactividad se desecharon las primeras sesiones grabadas. La selección de sesiones a codificar se hizo siguiendo el procedimiento de Blanco, Castellano y Hernández Mendo (2000).

El instrumento de codificación usado en la ULL fue el software AUGEN v. $\delta$ (Montero \& Montero, 2012), elaborado para el desarrollo de esta investigación. En la UdG, por dificultades técnicas de acceso al programa, se optó por una codificación tradicional de lápiz y papel.

\section{Análisis estadísticos}

Se calculó la fiabilidad entre observadores mediante la teoría de generalizabilidad (TG) y los patrones de comportamiento a través de análisis secuencial de retardos, con el programa SDIS-GSEQ, v. 5.1. (Bakeman \& Quera, 1996).

\section{Resultados}

Se realizaron se realizaron dos sesiones de fiabilidad correspondientes al 20\% de las sesiones codificadas, siguiendo el criterio de Patterson (1982), calculándola mediante la TG, obteniendo en ambos casos valores óptimos (coeficientes de generalizabilidad en la primera sesión entre 0.89 y 1 , y en la segunda entre 0.93 y 1$)$.

Como primer acercamiento a indicadores de buenas prácticas, se observó al profesorado en su desempeño en el aula, recogiendo mediante una escala de calificación los recursos empleados y los comportamientos mostrados por ellos, que se presentan en la tabla 2. En ella se señala con una cruz el uso de un recurso o la conducta realizada.

El profesorado se apoya en distintos materiales para dar sus clases: presentaciones multimedia, apuntes, uso del puntero láser para señalar, la tradicional pizarra, o bien materiales adicionales propios del contenido de la docencia que está desarrollando, especialmente en clases prácticas.

En el profesorado de la ULL todos utilizan presentaciones multimedia. Esto se facilita dado a la dotación de recursos multimedia en todas las aulas. Los profesores hacen uso variado del espacio, algunos se desplazan por el salón, otros se quedan de 
pie, se sientan o combinan entre estas posibilidades. Todos miran a sus alumnos cuando explican, aunque algunos también se centran en la presentación o sus notas.

El profesorado de la UdG utiliza en menor medida las presentaciones multimedia, por o general las aulas no cuentan con esta dotación. Algunos profesores centran la clase en la exposición del alumnado. En ocasiones hacen uso del móvil con fines distintos a la docencia. La posición en que dan las clases no presenta un patrón general tal como ocurre con el profesorado de la universidad canaria. Asimismo, todos acostumbran mirar al alumnado y casi la mitad de profesores se dirigen a ellos por su nombre. Esta práctica se ve promovida por la obligatoriedad de pasar lista de asistencia al inicio de clase.

Los indicadores de buenas prácticas directamente observables no se limitan a aspectos como los reseñados anteriormente, también supone patrones

TABLA 2.

Materiales y comportamientos del profesorado

\begin{tabular}{|c|c|c|c|c|c|c|c|c|c|c|c|}
\hline Profesores ULL & & 1 & 2 & 3 & 4 & 5 & 6 & 7 & 8 & 9 & 10 \\
\hline \multicolumn{12}{|l|}{ Materiales } \\
\hline Power Point & & $\mathrm{x}$ & $\mathrm{x}$ & $\mathrm{x}$ & $\mathrm{x}$ & $\mathrm{x}$ & $\mathrm{x}$ & $\mathrm{x}$ & $\mathrm{x}$ & $\mathrm{x}$ & $\mathrm{x}$ \\
\hline Notas & & -. & $\ldots$ & $\mathrm{x}$ & $\mathrm{x}$ & $\ldots$ & $\ldots$ & -. & -. & $\mathrm{x}$ & -. \\
\hline Puntero láser & & $\mathrm{x}$ & -. & -. & -. & $\mathrm{x}$ & -. & -. & $\ldots$ & $\ldots$ & -. \\
\hline Pizarra & & .. & .- & $\ldots$ & -. & -. & $\mathrm{x}$ & $\mathrm{x}$ & .. & -. & $\mathrm{x}$ \\
\hline Otros materiales & & .. & .. & .. & .. & .. & .. & .- & .. & $\mathrm{x}$ & $\mathrm{x}$ \\
\hline Lee de apuntes o portátil & & -. & -. & $\mathrm{x}$ & -. & -. & -. & -. & -. & -. & - \\
\hline \multicolumn{12}{|l|}{ Posición } \\
\hline De pie & & $\mathrm{x}$ & $\mathrm{x}$ & $\mathrm{x}$ & $\mathrm{x}$ & $\mathrm{x}$ & $\mathrm{x}$ & $\mathrm{x}$ & - & $\ldots$ & $\mathrm{x}$ \\
\hline Se desplaza & & $\ldots$ & $\ldots$ & $\ldots$ & $\ldots$ & $\ldots$ & $\mathrm{x}$ & $\mathrm{x}$ & $\ldots$ & $\mathrm{x}$ & - \\
\hline Sentado & & $\ldots$ & $\mathrm{x}$ & $\mathrm{x}$ & $\ldots$ & $\ldots$ & $\ldots$ & -. & $\mathrm{x}$ & $\ldots$ & - \\
\hline \multicolumn{12}{|l|}{ Mirada } \\
\hline Al alumnado & & $\mathrm{x}$ & $\mathrm{x}$ & $\mathrm{x}$ & $\mathrm{x}$ & $\mathrm{x}$ & $\mathrm{x}$ & $\mathrm{x}$ & $\mathrm{x}$ & $\mathrm{x}$ & $\mathrm{x}$ \\
\hline Otros sitios & & $\mathrm{x}$ & -. & $\mathrm{x}$ & $\mathrm{x}$ & $\mathrm{x}$ & - & -. & -. & -. & - \\
\hline Llama por el nombre & & -. & - & -. & -. & -. & $\mathrm{x}$ & -. & -. & $\mathrm{x}$ & -. \\
\hline Profesores UdG & 1 & 2 & & 3 & & 4 & 5 & & 6 & 7 & \\
\hline \multicolumn{12}{|l|}{ Materiales } \\
\hline Power Point & $\mathrm{x}$ & - & & $\mathrm{x}$ & & $\mathrm{x}$ & - & & - & $\mathrm{x}$ & \\
\hline Exposición estudiantes & -. & -. & & -. & & -. & -. & & $\mathrm{x}$ & $\mathrm{x}$ & \\
\hline Notas & -. & -. & & -. & & -. & -. & & -. & - & \\
\hline Pizarra & -. & -. & & -. & & $\mathrm{x}$ & -. & & -. & 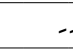 & \\
\hline Materiales & -. & .. & & $\mathrm{x}$ & & $\mathrm{x}$ & .- & & .- & - & \\
\hline Lee & $\mathrm{x}$ & - & & -. & & $\mathrm{x}$ & - & & - & 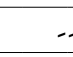 & \\
\hline Dispositivos no docentes & -. & - & & -. & & - & - & & $\mathrm{x}$ & $\mathrm{x}$ & \\
\hline \multicolumn{12}{|l|}{ Posición } \\
\hline De pie & $\mathrm{x}$ & $\mathrm{x}$ & & $\mathrm{x}$ & & $\mathrm{x}$ & - & & 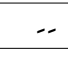 & 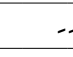 & \\
\hline Se desplaza & - & $\mathrm{x}$ & & - & & $\mathrm{x}$ & - & & $\mathrm{x}$ & 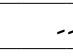 & \\
\hline Sentado & $\mathrm{x}$ & $\ldots$ & & $\ldots$ & & $\mathrm{x}$ & $\mathrm{x}$ & & $\mathrm{x}$ & $\mathrm{x}$ & \\
\hline \multicolumn{12}{|l|}{ Mirada } \\
\hline Al alumnado & $\mathrm{x}$ & $\mathrm{x}$ & & $\mathrm{x}$ & & $\mathrm{x}$ & $\mathrm{x}$ & & $\mathrm{x}$ & $\mathrm{x}$ & \\
\hline Otros sitios & - & $\ldots$ & & $\ldots$ & & $\mathrm{x}$ & - & & -. & $\mathrm{x}$ & \\
\hline Llama por el nombre & - & $\ldots$ & & - & & $\mathrm{x}$ & $\mathrm{x}$ & & $\mathrm{x}$ & 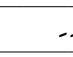 & \\
\hline
\end{tabular}

Fuente: elaboración propia 
de comportamiento tales como dar retroalimentación al responder a preguntas del alumnado, reforzar su participación o tras la explicación de contenido, fomentar la participación de sus estudiantes.

Cuando se evalúa utilizando el análisis secuencial de retardos de Bakeman y Quera (1996), la metodología observacional permite detectar patrones de comportamiento (Sackett, 1979) que reflejan un continuo de forma ordenada en el tiempo, destacando la persona focal objeto de estudio -en este caso el profesor-, así como la interacción con sus alumnos. Se analizan las relaciones de contingencia temporal entre conductas, permitiendo develar las reglas que regulan su mecánica interna, lo cual determina cómo cambian las probabilidades de ocurrencia de conductas en función de otras que se hayan presentado con anterioridad, apresando patrones de conductas estadísticamente significativos.

En el análisis de secuencia de retardos se elige una conducta antecedente a partir de la cual se contabilizan las veces que las otras conductas denominadas consecuentes, la siguen en orden para el primer retardo. En este estudio se consideró como conducta de criterio toda aquella con una frecuencia relativa superior a 0.02 , tomando como criterio cualquiera con frecuencia superior a cero.
Se registra el comportamiento tanto del profesor como de sus alumnos.

\section{Patrón de comportamiento referido a la retroalimentación al alumnado}

Cuando el alumno interviene, la adecuada retroalimentación del profesor es un indicador de buenas prácticas. Se recogen en este estudio dos patrones de comportamiento. Cuando el alumno participa por iniciativa propia, participación del estudiante (PE), seguido por la respuesta del profesor, respuesta al alumno (RA); y cuando el profesor comenta la respuesta del alumno como réplica a la pregunta del docente, correspondiente al patrón responde al profesor (RP) - respuesta al alumno (RA). Dichos patrones se presentan en la tabla 3 en la que el dígito de la izquierda indica las veces que la conducta consecuente sigue a la antecedente, y el de la derecha, las veces que se emite la consecuente.

Como se puede comprobar, siempre que el alumnado hace una intervención, sea pregunta o comentario, todo el profesorado, sin excepción, responde. La participación puede ser muy alta, como en las clases teóricas del profesor número 9 de la ULL, o pequeña (clases teóricas de los profesores 1, 2 y

TABLA 3.

Patrones relativos a la retroalimentación dada por el profesor

\begin{tabular}{|c|c|c|c|c|c|c|c|c|c|c|c|c|c|}
\hline \multicolumn{14}{|c|}{ Participación del estudiante (PE) - Responde al alumno (RA) } \\
\hline \multicolumn{14}{|c|}{ ULL } \\
\hline Profesor & 1 & 2 & 3 & & 4 & & & 6 & & & 8 & 9 & 10 \\
\hline Teoría & $4 / 6$ & $4 / 7$ & $5 / 5$ & & $3 / 11$ & & & $3 / 27$ & & & $7 / 12$ & $122 / 133$ & $45 / 51$ \\
\hline Prácticas & $27 / 36$ & .. & $24 / 2$ & & $6 / 13$ & & & $2 / 4$ & $84 /$ & & $16 / 26$ & $43 / 50$ & $74 / 80$ \\
\hline \multicolumn{14}{|c|}{ UdG } \\
\hline \multirow{2}{*}{\multicolumn{2}{|c|}{ Profesor }} & & & & 2 & & & 4 & 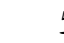 & & 6 & 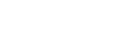 & \\
\hline & & & & & 8/35 & & & $59 / 66$ & 15 & & $14 / 26$ & 21 & \\
\hline \multicolumn{14}{|c|}{ Responde al profesor (RP) - Responde al alumno (RA) } \\
\hline \multicolumn{14}{|c|}{ ULL } \\
\hline Profesor & 1 & 2 & & 3 & & & 5 & & 5 & 7 & 8 & 9 & 10 \\
\hline Teoría & -. & .. & & 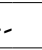 & & & -. & & 27 & $4 / 6$ & - & .. & -. \\
\hline Prácticas & - & $2 / 2$ & & - & & & $7 / 10$ & & - & -. & - & -. & -. \\
\hline \multicolumn{14}{|c|}{$\mathrm{UdG}$} \\
\hline \multirow{2}{*}{\multicolumn{2}{|c|}{ Profesor }} & & & & 2 & & & 4 & & & 6 & 7 & \\
\hline & & & & & .. & & & .. & & & $10 / 26$ & .. & \\
\hline
\end{tabular}

Fuente: elaboración propia 
3 de la ULL). Solo hay nula participación en las clases teóricas del profesor número 7. En la UdG todos los profesores contestan a las respuestas de su alumnado, siendo el patrón de comportamiento más homogéneo, lo que indica que la participación de los alumnos es más común en esta universidad.

Como se espera, el segundo patrón es menos frecuente que el anterior, pues mientras que en el primer caso el docente responde a una intervención de un alumno, el patrón que se está analizando aquí es menos relevante en cuanto a retroalimentación, ya que no supone la réplica a una pregunta sino un comentario adicional.

\section{Patrones relacionados con el refuerzo} a la participación del alumnado

Los patrones relacionados con la emisión de refuerzos son también de dos tipos: ante las intervenciones del alumnado por propia iniciativa o a manera de respuesta. Todos los profesores refuerzan la participación de sus alumnos por propia iniciativa o como respuesta al profesor, tanto en clases teóricas como prácticas. Ahora bien, el número de refuerzos emitidos difiere de forma relevante: desde 3 hasta 78 .
En el caso de los profesores de la UdG, todos aplican refuerzo, aunque su frecuencia no es muy alta , tampoco parece haber diferencia en los refuerzos a estos dos tipos de conductas, salvo en la profesora 4 que es quien más refuerza, pero solo las respuestas de los alumnos a sus preguntas, y en el profesor 3 que en cambio, refuerza la participación del estudiante por iniciativa propia.

\section{Patrones referidos al fomento de la participación}

Otro indicador de buenas prácticas docentes es que el profesor anime a sus alumnos a participar en clase como una conducta consecuente, donde esta sigue a la conducta previa con una probabilidad superior a la que se espera por azar, en los patrones explicación del profesor (EP) - fomento de la participación (FP) y en responde al profesor (RP) - fomento de la participación (FP). También como conducta antecedente, formando parte del patrón fomento de la participación (FP) - responde al profesor (RP). Como se puede apreciar en la tabla número 5 , el fomento de la participación sigue en la mayoría de los casos a la explicación que da el profesor, lo cual coonstituye una clara muestra de buenas prácticas porque

\section{TABla 4.}

Patrones relacionados con el refuerzo del profesor (ULL)

ULL

\begin{tabular}{|c|c|c|c|c|c|c|c|c|c|c|}
\hline \multicolumn{11}{|c|}{ Participación del estudiante (PE) - Refuerzo (RF) } \\
\hline Profesor & 1 & 2 & 3 & 4 & 5 & 6 & 7 & 8 & 9 & 10 \\
\hline Teoría & -. & $4 / 7$ & $2 / 4$ & $3 / 5$ & -. & $7 / 64$ & - & $2 / 3$ & - & -. \\
\hline Prácticas & $6 / 8$ & -. & $12 / 16$ & -. & $3 / 10$ & -. & -. & $13 / 19$ & -. & $13 / 25$ \\
\hline \multicolumn{11}{|c|}{ UdG } \\
\hline \multirow[t]{2}{*}{ Profesor } & \multicolumn{2}{|c|}{1} & 2 & \multicolumn{2}{|c|}{3} & 4 & 5 & 6 & 7 & \\
\hline & \multicolumn{2}{|c|}{$7 / 17$} & $4 / 14$ & \multicolumn{2}{|c|}{$16 / 30$} & -. & $3 / 8$ & $3 / 6$ & $5 / 10$ & \\
\hline \multicolumn{11}{|c|}{ Responde al profesor (RP) - Refuerzo (RF) } \\
\hline \multicolumn{11}{|c|}{ ULL } \\
\hline Profesor & 1 & 2 & 3 & 4 & 5 & 6 & 7 & 8 & 9 & 10 \\
\hline Teoría & .. & -. & -. & $2 / 5$ & $27 / 31$ & $48 / 64$ & $31 / 42$ & -. & $58 / 78$ & $60 / 82$ \\
\hline Prácticas & .. & $3 / 3$ & -. & $6 / 7$ & -. & -. & $54 / 66$ & $6 / 19$ & $53 / 68$ & $12 / 25$ \\
\hline \multicolumn{11}{|c|}{ UdG } \\
\hline Profesor & \multicolumn{2}{|c|}{1} & 2 & \multicolumn{2}{|c|}{3} & 4 & 5 & 6 & 7 & \\
\hline & \multicolumn{2}{|c|}{$8 / 17$} & $5 / 14$ & \multicolumn{2}{|c|}{.. } & $20 / 40$ & -. & -. & -. & \\
\hline
\end{tabular}

Fuente: elaboración propia 
incentiva la participación del alumnado. En cambio solo aparece fomento de la participación tras la conducta del alumno responde al profesor (RP) en 4 de los 10 profesores de la ULL, y en todos los casos del profesorado de la UdG. Se puede afirmar que hay dinamismo en las clases, pues el fomento de la participación va seguido, casi siempre, de la respuesta del alumnado.

\section{Discusión}

Las políticas de calidad juegan un papel relevante en la educación a todos los niveles, especialmente en la educación superior. Esto conlleva la búsqueda de indicadores que permitan mostrar la existencia de dicha calidad, así como la amplia gama de procedimientos que permiten medirla de una u otra forma (Martínez, 2013).

TABLA 5.

Patrones relativos a promover la participación del alumnado
Las buenas prácticas docentes constituyen una forma de entender la calidad y presenta indicadores claros, tiene una perspectiva multimodal, como se puede desprender de cómo la caracterizan los autores que se han dedicado a estudiarla (Bain, 2006; Imbernón, 2013; Marqués, 2002). Algunos indicadores se comprueban con los contenidos que el docente imparte, otros corresponden a aspectos actitudinales, como la fe en el 'buen hacer' y la responsabilidad del alumnado, otros tienen que ver con la evaluación. Una gran cantidad de los indicadores de buenas prácticas son comportamientos que se realizan en el aula y por lo tanto, son directamente observables.

La calidad conlleva evaluación necesariamente, requiere comprobar que se presenten los indicadores de tal calidad. Por ello es imprescindible crear procedimientos de medida que permitan determinar

ULL

Explicación del profesor (EP) - Fomento de la participación (FP)

\begin{tabular}{lcccccccccc}
\hline Profesor & 1 & 2 & 3 & 4 & 5 & 6 & 7 & 8 & 9 & 10 \\
\hline Teoría & - & $22 / 50$ & -- &.- & $50 / 124$ & $117 / 172$ & $32 / 69$ & $2 / 3$ & $79 / 239$ & $74 / 251$ \\
\hline Prácticas & - & - & -- &.- & $20 / 55$ & - & $61 / 179$ & $10 / 26$ & $36 / 72$ & $49 / 91$ \\
\hline UdG & & & & & & & & & & \\
\hline Profesor & 1 & & 2 & 3 & 4 & 5 & 6 & 7 & \\
\hline & $89 / 206$ & & $41 / 127$ & $25 / 75$ & $45 / 162$ & $38 / 106$ & $54 / 162$ & $20 / 82$ & $89 / 206$ \\
\hline
\end{tabular}

ULL

Responde al profesor (RP) - Fomento de la participación (FP)

\begin{tabular}{|c|c|c|c|c|c|c|c|c|c|c|}
\hline Profesor & 1 & 2 & 3 & 4 & 5 & 6 & 7 & 8 & 9 & 10 \\
\hline Teoría & -. & .- & -. & -. & -. & -. & $37 / 42$ & -. & 26/239 & .- \\
\hline Prácticas & -. & -. & -. & -. & $5 / 10$ & -. & $38 / 179$ & $11 / 26$ & 25/169 & -. \\
\hline \multicolumn{11}{|l|}{$\mathrm{UdG}$} \\
\hline Profesor & \multicolumn{2}{|c|}{1} & \multicolumn{2}{|c|}{2} & 3 & 4 & 5 & 6 & 7 & \\
\hline & \multicolumn{2}{|c|}{$69 / 206$} & \multicolumn{2}{|c|}{$35 / 127$} & $18 / 75$ & $56 / 162$ & $40 / 106$ & $56 / 162$ & $17 / 82$ & \\
\hline
\end{tabular}

ULL

Fomento de la participación (FP) - Responde al profesor (RP)

\begin{tabular}{|c|c|c|c|c|c|c|c|c|c|c|}
\hline Profesor & 1 & 2 & 3 & 4 & 5 & 6 & 7 & 8 & 9 & 10 \\
\hline Teoría & .. & $4 / 4$ & -. & $6 / 10$ & $5 / 21$ & $135 / 152$ & $53 / 60$ & .. & $172 / 183$ & $138 / 150$ \\
\hline Prácticas & $5 / 12$ & -. & $3 / 9$ & $16 / 20$ & $30 / 36$ & -. & $148 / 161$ & $23 / 26$ & $119 / 131$ & $45 / 46$ \\
\hline \multicolumn{11}{|l|}{ UdG } \\
\hline Profesor & \multicolumn{2}{|c|}{1} & \multicolumn{2}{|c|}{2} & 3 & 4 & 5 & 6 & \multicolumn{2}{|l|}{7} \\
\hline & \multicolumn{2}{|c|}{$163 / 179$} & \multicolumn{2}{|c|}{$114 / 116$} & $62 / 71$ & $123 / 139$ & $82 / 87$ & $141 / 145$ & $49 / 51$ & \\
\hline
\end{tabular}

Fuente: elaboración propia 
que el profesorado realiza o no, buenas prácticas docentes. Como se presentó en este estudio, algunos indicadores son muy sencillos y se pueden recoger con una simple observación a través de una escala de calificación, señalando si un determinado aspecto, que señala buena o mala práctica, se da.

El mayor énfasis en modelos educativos donde el alumnado es protagonista de su formación, prima el enfoque de enseñanza-aprendizaje, esto hace que no se pueda aislar la conducta del profesor, importa sustancialmente la interacción que mantiene con su alumnado. El empleo de la metodología observacional con los análisis específicos, permiten apresar tanto el comportamiento del profesorado, como -y esto resulta especialmente relevante en el caso de buenas prácticas-, su forma de interactuar con los alumnos. El análisis secuencial de retardos es un instrumento analítico que permite gran precisión. Además, en la medida en que determina claramente los patrones conductuales, sirve a los objetivos de una evaluación productiva, es decir que posibilita recomendar mejoras claramente objetivadas, para aquel profesorado que deba fortalecer sus buenas prácticas docentes.

Con el instrumento aquí presentado se pueden estudiar algunos indicadores de buenas prácticas docentes. Otros no directamente observables, precisan el uso de metodologías distintas, como autoevaluaciones de los profesores y estudiantes. No obstante, quedan comportamientos que también son observables, que no se pueden recoger con este instrumento, como puede ser la forma en que el profesor se dirige al alumno, mostrando aceptación, respeto o todo lo contrario. Será preciso por tanto, seguir creando instrumentos de medida, escalas o instrumentos de observación que permitan estudiar de forma exhaustiva las buenas prácticas docentes.

Por otra parte, la muestra que se ha estudiado aquí, aunque recoge dos entornos educativos distintos, es pequeña, como es habitual en metodología observacional, por lo que es preciso realizar estudios similares con otras muestras de profesorado universitario. Sin embargo, el procedimiento de medida que se presenta permite objetivar de forma clara, comportamientos docentes de interacción que señalan buenas prácticas docentes y comportamientos objeto de mejoras. De una manera u otra, permite analizar y modificar si corresponde, buenas prácticas docentes.

\section{Referencias}

Anguera, M. T. (2008). Evaluación de programas desde la metodología cualitativa. Acción Psicológica, 5(2), 87-101.

Anguera, M. T., Blanco, A., \& Losada, J. L. (2001). Diseños observacionales, cuestión clave en el proceso de la metodología observacional. Metodología de las Ciencias del Comportamiento, 3(2), 135-160.

Anguera M. T., Blanco, A., Losada, J. L., \& Hernández, A. (2000). La metodología observacional en el deporte: conceptos básicos. Revista Digital Efdeportes, 5(24). Recuperado de: http://www.efdeportes.com/ efd24b/obs.htm

Bain, K. (2006). Lo que hacen los mejores profesores universitarios. Valencia: Publicacions de la Universitat de Válencia.

Bakeman, R., \& Quera, V. (1996). Análisis de la interacción. Análisis secuencial con SDIS y GSEQ. Madrid: Ra-Ma.

Blanco, A., Castellano, J., \& Hernández Mendo, A. (2000). Generalizabilidad de las observaciones en la acción del juego en el fútbol. Psicothema, suplemento, 12(Supl. 2), 81-86.

Cadenas, M., Rodríguez, M., \& Díaz, M. (2012). Los equipos de entrenamiento: una muestra para el estudio de los sesgos en la fiabilidad entre parejas de observadores. Revista de Investigación y Divulgación en Psicología y Logopedia, 2(2), 41-46.

Cid-Sabucedo, A., Pérez-Abellás, A., \& Zabalza, M. (2009). Las prácticas de enseñanza declaradas de los "mejores profesores" de la universidad de Vigo. RELIEVE, 15(2), 1-29. Recuperado de: http://www. uv.es/RELIEVE/v15n2/RELIEVEv15n2_7.htm

Gaitan Riveros, C., Campo Vasquez, R., Garcia Cano, L., Granados, L. F., Jaramillo Pabon, J., \& Panqueva Tarazona, J. (2005). Prácticas educativas y procesos de formación en la educación superior. Bogota: Pontificia Universidad Javeriana.

Imbernón (2013). Estrategias de participación en el aula. Curso impartido en la Universidad de La Laguna, España. 
Marqués, P. (2002). Buenas Prácticas Docentes. Revista DIM, 19. Recuperado de: http://peremarques.pangea.org/bpracti.htm.

Martínez, J. F. (2013). Combinación de las mediciones de la práctica y el desempeño docente: consideraciones técnicas y conceptuales para la evaluación docente. Pensamiento Educativo. Revista de Investigación Educacional Latinoamericana, 50(1), 4-20. doi: http://dx.doi.org/10.7764/PEL.50.1.2013.2

Montero, J., \& Montero, J. (2012). Programa Software Augen, v. $\delta$. Computer Business Solutions.

Patterson, G. R. (1982). Coercive family process. Eugene, OR: Castalia.
Rodríguez, M., Cadenas, M., \& Díaz, M. (2011). Estimación del tiempo de registro óptimo para la observación de las funciones docentes del profesor universitario. Revista de Investigación y Divulgación en Psicología y Logopedia, 1(1), 10-15.

Rodríguez-Naveiras, E. (2011). PROFUNDO: Un instrumento para la evaluación de proceso de un programa de altas capacidades. Tesis Doctoral no publicada. Universidad de La Laguna, España.

Sackett, G. P. (1979). The lag sequential analysis of contingency and ciclicity behavioral interaction research. En J. Osofsky (Ed.). Handbook of infant development (pp. 623-649). Nueva York: Wiley. 